\title{
The study on the attitudes of people with different ages towards transgenic food
}

\author{
Aizhu Liu ${ }^{1}$ \\ ${ }^{1}$ Maple leaf international school, Teda, Tianjin, 300450, China
}

\begin{abstract}
With the rapid development of contemporary society, increasing number of people begin to pay attention to whether the food they eat are healthy enough. Under such considerable population, the traditional method of growing crops becomes inadequate to meet the needs of the population. As a consequence, scientists invent a new way to grow food called genetic modification. This paper is to investigate the attitudes of people with different ages to GM foods. In order to reach that goal, it conducts a questionnaire survey in Tianjin, China. Serving as the results, the data gathered show that a large part of population older than 40 have a skeptical attitude toward transgenic food and, on the contrary, most of the people younger than 40 hold an optimistic attitude toward the future of transgenic food. In conclusion, the development of GM foods are promising in this city.
\end{abstract}

\section{Introduction}

As known to the general public, molecular biotechnology is used to transfer the genes of one organism to another species so that the characteristics of the species can be changed in the desired direction, which is called "transgenic technology". (Channabasavaiah B. Gurumurthy, 2020)[1]. The crops cultivated by transgenic technology are called "transgenic crops". Genetically modified crops as direct food or as raw materials to produce food is "genetically modified food". Due to its characteristic of that, it can be largely produced, so transgenic crops play a critical role in the maintenance of the world' s population. Recently, with the rapid spread of GM crops, general public begins to raise more concern about its safety. The focus of this paper is to investigate general public's attitude about transgenic food as well as GM crops' development prospect in Tianjin. To be specific, this paper attempts to show common people's recognization about genetic modification technology and their acceptability of this kind of food. This investigation adopts questionnaire to collect the data, and there are 100 people in the test group. And this crowd includes 50 females and 50 males with various ages.

This research provides a new insight into the future development of GM crops as well as changes people' $\mathrm{s}$ perspective about this kind of crops. Understanding the link between genetic modification crops and general customers enables scientist to promote GM technology. Therefore, they can make it more suitable and acceptable for human, and brings human more benefits. For example, a new generation of genetically modified crops with more nutritional component will be popular among consumers.

\section{Experiment details and analysis}

When mentioning to the safety of the GM crops, according to the Food and Agriculture Organization of the United Nations (FAO), World Health Organization (WTO) and WFP (Jingfei Guo, Kanglai He, Richard L. Hellmich, 2016), "GM foods currently available on the international market have passed risk assessments and are unlikely to pose a risk to human health [6]. And, in the general population in the countries where they are approved, no human health effects have been found from the consumption of these foods." These groups confirm that no scientific evidence has been found to suggest that eating these foods has negative effects on human health.

Many researchers have utilized questionnaires to figure out respondents' thoughts. An excellent questionnaire should not only reflect the purpose of the respondents correctly, but also convey their thoughts accurately. After collecting the questionnaires, the surveyors sort out the answer of questionnaires, and then analyze, and summarize the information therein. The quality of the questionnaire directly affects the quality of the survey results and the survey report. Questionnaire plays a central role in the research process.

As constructing questionnaires is a common method in the study of scientific and sociology related topics. There are many previous examples about using questionnaires as a form of scientific investigation about the public understanding of HIV/Aids. Kadesha (2008) employs a questionnaire including 30 questions to analyze the public understanding of HIV [2]. The effects are significant. It accurately points out that there are a 
large amount of people in the world who are not familiar with this disease, not to mention knowing how to prevent it. Therefore it becomes the reason why this study chooses to construct a questionnaire including 20 questions to test the respondent's attitudes towards GM crops.

Obviously, questionnaires are not the only method of researching this topic. Most researchers investigating transgenic technique have utilized feeding trials (Chunmiao Fan, Fengci Wu, 2019): a technology of livestock production, by translating the results into livestock and poultry productivity and benefits of the important links, and applying in the promotion work, the results of the experiment could be directly demonstrated and popularized in the large fields under similar production conditions [3]. A research called "GRACE" (GMO Risk Assessment and Communication of Evidence), an authoritative experiment among the world. After a series of experiments, these researchers confirms that the GM crops currently on market is completely harmless.

However, questionnaires survey is particularly useful in studying the crowds' attitudes, which means, it becomes extremely suitable for the main topic of this essay. The design of the questionnaire is based on the characteristics of GM crops (Calestous Juma Katherine Gordon, 2014) [4].100 people randomly chosen from Tianjin are recruited in this study. They are different in gender and age. Group one includes 50 people who are younger than 40 and group two elects 50 people older than 40 . The reason of the classification of these two age groups is that people older than 40 , will pay more attention to their health and begin to choose their diet based on the safety of foods (Cong Liu, Xiaojiu Li, Muhui Lin, Limin Zheng \& Xiaohong Chen, 2020) [3]. In this way, it is easy for this research to infer their attitude of GM foods.

The following are the questions in questionnaires:

1.Your gender: [single choice] female / male

2.Your age: [single choice] 40- 40+

3.Your education: [single choice] junior high school / High School and Junior College /Undergraduate course /Post graduate/ PHD

4.Your monthly income: [multiple choice] 0-2000/ 2000-5000 / 5000-10000 / 10000+Yuan

5.Your current occupation: [single choice] farmers/ workers in enterprises/ public institutions personnel/ private business owners / students

6.Before this survey, did you hear about GM foods? [single choice] Yes. / no.

7.What's your attitude towards GM food? [single choice]

Positive / Skeptical / I don't care.

8.How did you learn about GM foods? [Multiple choice]

$\mathrm{TV}$ / magazine / network / professional food safety organization publicity / teachers, family friends / other

9.Do you think the following foods are genetically modified food? [Multiple choice]

$\mathrm{KFC}$ / purple potato / oreo / oil / cherry tomato / dried bean curd
10. What do you think is true about gm foods (Hui-Ru Jia, Li-Li Geng, 2016)[6]? [multiple choice]

- There is no difference between GM food and hybrid crops.

- Scientific research on GM food is still incomplete and should be treated with caution.

- GM food has been circulating in the international market for many years and has entered everyone's life, which proves that kind of food is safe and harmless.

- There are already more than 3,000 GM foods on the North American market, and GM food are approved for consumption in each country.

11. What do you think are the advantages of GM food? [Multiple choice]

- Full of nutrition that the human need.

- More delicious than the traditional food.

- Lower price, and sufficient production.

- Environmental friendly.

- Other.

12. What do you think are the disadvantages of GM food? [Multiple choice]

- Lower nutritional value than non-GM foods.

- Prone to potentially unknown threats to the body.

- It may destroy the ecological balance.

- It may violate the laws of natural development and may cause food security problems.

- Other

13. What is your attitude towards buying GM food? [Multiple choice]

- I will buy GM food.

- It doesn't matter. It just depends on the price.

- I don't buy GM food, but I don't object to it.

- I don't buy GM food and I'm against it.

14. How often do you eat GM food? [single choice]

-Very often.

-Occasionally.

-Impossible to buy.

15. What GM foods do you think are currently available in the Chinese market? [Multiple choice]

- bean products

- dairy

- Pork, beef and other meats

- Fungi, such as mushrooms

-Rice, corn and other staple foods.

- cooking oil

- All kinds of fish and aquatic products.

-Vegetables, fruits

-None of the above

16.Do you support the research and promotion of GM food in China? [Single choice]

-Support -Reject -Doesn't matter

17. What steps do you think the government should take? [Single choice]

-Completely forbidden. Formulate relevant policies and regulations on GM food, and prohibit the production or import of GM food.

-Strict safety management and approval system. Strictly restrict the production and import of GM food, and control or restrict breeding areas for gm plants.

-Strengthen the guidance and publicity of safety risks and identification methods, and specially marked GMFS to ensure the public to know their rights and options. 
18. What do you think of the future of GM food? [Single choice]

-A large market, which may completely replace the traditional food.

-It is not accepted by consumers and squeezed out of the market by traditional food.

-There is a common consumer market with traditional food for consumers to choose freely.

In order to identify their attitudes, the participants are asked to complete these 18 questions. Then this paper utilizes Excel to analyze those data collected. This data set is quite revealing in several ways. And the answer of this questionnaire is presented as follow.

First, the answers of question 6 shows that there are as more as $99 \%$ participants have heard about transgenic foods, and for question 7,81 percent of the respondents younger than 40 show positive attitudes while about 89 percent of the people older than 40 show skeptical attitudes as shown in Figure 1.

\section{Different attitudes from people with different ages towards GM food}

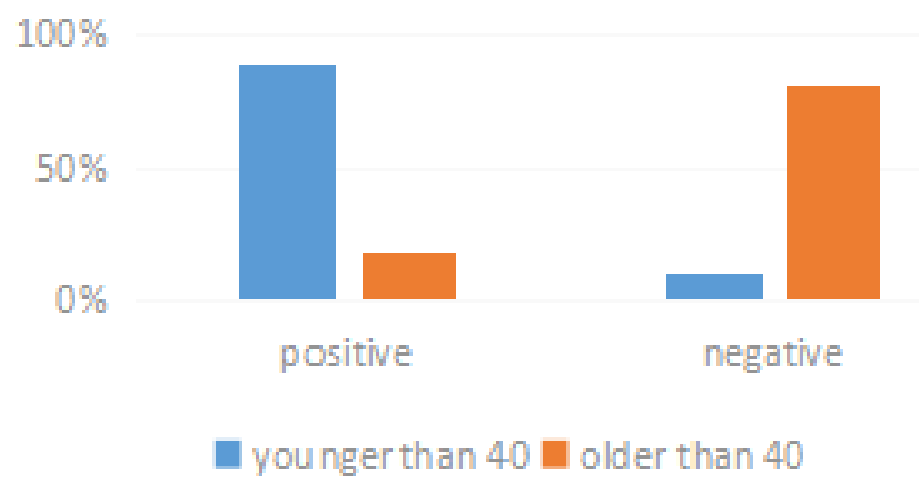

Fig 1. Different attitudes from people with different ages towards GM food

In question 8 , there are $29 \% / 68 \%$ people that are very concern or know some information about GM crops. This result indicates that most of the common people in Tianjin have heard and concerned about GM technology. And, in question 7 , the results show that $67 \%$ people get in touch with the word " genetic modification food" through the internet and their family member, teachers, and friends. This is enough to prove the huge social influence of GM technology. Then in question 10, a large extent of people have thought that "Scientific research on GM food is still incomplete and should be treated with caution". However, there are still a small amount of people think that"There are already more than 3,000 gm foods on the North American market, and gm foods are approved for consumption in each country" which can prove its safety. As mentioned above, now there is enough scientific evidence that all the transgenic foods on market is safe enough. Therefore, this shows that ordinary people have more objectivity than subjectivity in judging the safety of genetically modified foods.

Next for question 11,12, people have been asked the advantages of transgenic foods. Almost two-thirds (64\%) of the participants report that they think GM foods can largely produced and benefits people's health in some aspects. On the other side, when talking to the disadvantage of GM foods, more than half of them (55\%) argue that it has potential safety problems and may even post threat to the human's healthy. For question $14,47 \%$ of them admit that they will buy transgenic foods and the other $31 \%$ admit that they won't buy transgenic foods but they don't mind others to buy it. Followed by question 15 , the majority of the participants thinks GM foods are not common but they sometimes eat it.

When discussing the future of transgenic foods, $97 \%$ of the survey takers support the research and promotion of GM food in China and they think the government should "provide more guidance and publicity of safety risks and identification methods, and specially marked GMFS to ensure the public to know their rights and options", at the same time, there has to be "strict safety management and approval systems". Overall, they contend an optimistic attitude about the future development of GM foods in China and prefer to build a common consumer market with traditional food for consumers to choose freely.

Through the analysis of the results, a possible explanation for the crowds' tendency might be that most of the people younger than 40 know GM from internet or taking biology classes, but they don't have a deep understanding with this technology. Most of the people older than 40 get in touch with GM foods through talking with their friends or colleagues. They may be affected by others and may have bias about GM technology. And this could be the reason why they think GM foods on market 
may post a potential threat to human's health. According to the final data, the more policies and guidance from government, the more professional ways they know transgenic foods. If the government can strive to improve the citizens' understanding and let them understand more scientific and professional knowledge of GM technique, there will be less doubt about GM food, and the future of GM food will be more promising.

\section{Conclusion}

The aim of this investigation is to explore different attitudes towards GM food from people younger and older than 40. This data is collected through a questionnaire including 20 various and comprehensive questions. The result confirms that people younger than 40 tend to hold an optimistic attitude toward GM food, while people older than 40 prefer to have a negative attitude toward GM food. However, most of prefer to build a common consumer market with traditional food for consumers to choose freely. In general, there is still a large extent of the participants who are lack of a correct understanding of GM technology, which make them think that there are potential problems on transgenic foods now in market. To resolve this issue, the government should introduce some polices to control GM food market to alleviate people's concern and therefore promote the optimistic attitudes of citizens as well as the future development of GM foods.

\section{Acknowledgement}

First, I really appreciate those 100 people who fill in the questionnaires and are willing to share their understanding with public. And I would like to show my sincere gratitude to my friends and teachers. After I received that much answers of questionnaires, it is my friend who help me to collect them and set them into different categories. Likewise, during the process of writing this essay, my teacher gives me a lot of suggestions and helps me to polish my word.

\section{References}

1. Schilit, Samantha L. P., et al. Pronuclear Injection-Based Targeted Transgenesis. Current Protocols in Human Genetics, 2016:1-28.

2. Kadesha. "HIV/AIDS Knowledgeable Quiz

Questions 1 - ProProfs Quiz.”

www.proprofs.com/quiz-school/story.php?title=hiva ids-quiz

3. Fan C, Wu F, Dong J, et al. No impact of transgenic crylle maize on the diversity, abundance and composition of soil fauna in a 2-year field trial[J], 2019, 9(1).

4. Juma, Calestous, and Katherine Gordon. Transgenic Crops and Food Security[J]. 2014: 45-58

5. Guo J, He K, Hellmich R L, et al. Field trials to evaluate the effects of transgenic crylle maize on the community characteristics of arthropod natural enemies[J]. entific Reports, 2016, 6(1):22102.

6. Jia H R, Geng L L, Li Y H, et al. The effects of Bt Crylle toxin on bacterial diversity in the midgut of Apis mellifera ligustica (Hymenoptera: Apidae)[J]. 2016, 6(1):24664. 